\title{
TOQUE TERAPÊUTICO COMO ESTRATÉGIA PARA REDUÇÃO DE ESTRESSE EM ESTUDANTES DE ENFERMAGEM
}

\section{THERAPEUTIC TOUCH AS STRATEGY FOR STRESS REDUCTION IN NURSING STUDENTS}

\section{TACTO TERAPÉUTICO COMO ESTRATEGIA PARA REDUCIR EL ESTRÉS EN ESTUDIANTES DE ENFERMERÍA}

\author{
Nerizane Cerutti Fornari ${ }^{1}$, Diéssica Roggia Piexak ${ }^{2}$, Aline Cristina Calçada de Oliveira ${ }^{3}$, \\ Daiane Porto Gautério Abreu ${ }^{4}$, Edison Luiz Devos Barlem ${ }^{5}$, Silvana Possani Medeiros ${ }^{6}$
}

\section{RESUMO}

Objetivo: Analisar o toque terapêutico como estratégia para redução de estresse em estudantes de enfermagem. Métodos: Estudo longitudinal do tipo caso-controle realizado com 20 estudantes de enfermagem. O instrumento de pesquisa foi uma escala de avaliação de estresse antes e após a aplicação de toque terapêutico. Para análise dos dados, utilizaram-se os testes de Wilcoxon e Kruskal-Wallis. Resultados: O toque terapêutico mostrou-se eficaz para redução do estresse nos domínios 1, 3 e 6 da escala, não sofrendo influência de fatores como atividade de lazer, renda, ter ou não filhos, e série atual. Conclusão: $O$ toque terapêutico foi eficiente na redução dos níveis de estresse, auxiliando os estudantes de enfermagem quanto ao enfrentamento do mesmo. Outros estudos são necessários para aprofundar pesquisas na área, que visem propiciar recursos a serem buscados pelos estudantes de enfermagem para minimizar o estresse vivenciado.

Descritores: Toque terapêutico; Estudantes de enfermagem; Estresse psicológico.

\begin{abstract}
Objective: To analyze the Therapeutic Touch as a stress reduction strategy in nursing students. Methods: A longitudinal study of case-control type, performed with 20 nursing students. The research instrument was a stress evaluation scale applied before and after the Therapeutic Touch application. For data analysis, the Wilcoxon and Kruskal-Wallis tests were used. Results: The Therapeutic Touch was shown to be effective in reducing stress in the 1, 3 and 6 areas of the scale, not influenced by factors such as leisure activity, financial income, having children or not, and current college semester. Conclusion: The therapeutic touch was effective in reducing stress levels, assisting nursing students in its coping. To deepen the research, other studies are required, aiming to provide resources to be sought by nursing students to minimize the stress experienced.
\end{abstract}

Descriptors: Therapeutic touch; Nursing students; Psychological stress.

\footnotetext{
${ }^{1}$ Enfermeira, Rio Grande, Rio Grande do Sul.

${ }^{2}$ Enfermeira. Doutora pelo Programa de Pós-Graduação em Enfermagem da Universidade Federal do Rio Grande.

${ }^{3}$ Doutora em Educação Ambiental, Docente da Escola de Enfermagem, Universidade Federal do Rio Grande FURG, Rio Grande, Rio Grande do Sul.

${ }^{4}$ Doutora em Enfermagem, Docente da Escola de Enfermagem, Universidade Federal do Rio Grande - FURG, Rio Grande, Rio Grande do Sul.

${ }^{5}$ Doutor em Enfermagem, Docente da Escola de Enfermagem, Universidade Federal do Rio Grande - FURG, Rio Grande, Rio Grande do Sul.

${ }^{6}$ Enfermeira. Mestrando do Programa de Pós-Graduação em Enfermagem da Universidade Federal do Rio Grande - FURG, Rio Grande, Rio Grande do Sul.
} 


\section{RESUMEN}

Objective: Analizar el Tacto Terapéutico como estrategia para reducir el estrés en estudiantes de enfermería. Métodos: Estudio longitudinal del tipo caso-control realizado con 20 estudiantes de enfermería. El instrumento de investigación fue una escala de evaluación de estrés antes y después de la aplicación del Tacto Terapéutico. Para el análisis de los datos, se utilizaron las pruebas de Wilcoxon y Kruskal-Wallis. Resultados: El Tacto Terapéutico se mostró eficaz para reducir el estrés en los dominios 1,3 y 6 de la escala, no sufriendo influencia de factores como actividad de ocio, renta, tener o no hijos, y semestre universitario actual. Conclusión: El Tacto Terapéutico fue eficiente en la reducción de los niveles de estrés, auxiliando a los estudiantes de enfermería en cuanto al enfrentamiento del mismo. Otros estudios son necesarios para profundizar investigaciones en el área que apunten a propiciar recursos a ser buscados por los estudiantes de enfermería para minimizar el estrés vivido.

DESCRIPTORES: Tacto Terapéutico; Estudiantes de enfermería; Estrés Psicológico.

\section{INTRODUÇÃO}

As pressões geradoras de estresse são vivenciadas na vida pessoal, social e profissional, e isso não é menos diferente durante o período de formação universitária. ${ }^{1} \mathrm{O}$ estresse, como reação inespecífica do organismo diante de pressões exercidas sobre o sistema orgânico, pode desencadear sintomas como ansiedade, tensão, angústia, insônia, alienação, dificuldades interpessoais, dúvidas quanto a si, dificuldade de relaxar, ira e hipersensibilidade emotiva. ${ }^{2-4}$

Durante o processo de formação, o estudante de enfermagem enfrenta diversas situações de crise e pode vivenciar sentimentos distintos. Além de estar em um momento ímpar de sua vida, buscando se profissionalizar para encontrar seu lugar no mercado de trabalho, também está exposto ao processo de saúde/doença de seus clientes, sendo passível de convivência com o sofrimento, as doenças terminais, os conflitos familiares e a morte. ${ }^{4}$

Estudo que objetivou analisar a ocorrência da investigação científica sobre a saúde mental do aluno do curso de graduação em enfermagem, no Brasil, nos últimos 21 anos, verificou que entre os momentos críticos podem-se destacar: a iniciação do estudante no meio acadêmico, ambiente com o qual ainda não está familiarizado; o início das atividades práticas, nas quais os estudantes se envolvem com situações complexas do sistema de saúde e com a sua própria insegurança; e o término do curso, quando o estudante, na maioria das vezes, preocupa-se com o mercado de trabalho e as exigências da profissão. Frente a esses momentos, iniciativas que possam vir a apoiá-los no enfrentamento das suas dificuldades são imprescindíveis. ${ }^{5}$

No entanto, mesmo compreendendo que o processo de formação do enfermeiro apresente diversas situações que 
desencadeiam estresse e ansiedade, não se verifica a oferta de estratégias que fortaleçam emocionalmente os estudantes para esse enfrentamento, exigindo estudos com essa abordagem. ${ }^{6}$

É neste contexto que emergem as práticas integrativas e complementares, como possibilidades de instrumentalização dos estudantes de enfermagem para o enfrentamento de situações de estresse. ${ }^{7}$

As terapias integrativas e complementares são reconhecidas pelo Conselho Federal de Enfermagem (COFEN), na Resolução COFEN-389, de 18 de outubro de 2011, como especialidade do Profissional de Enfermagem. ${ }^{8}$

Dentre as práticas integrativas e complementares, enfatiza-se o Toque Terapêutico, que teve início com a enfermeira norte-americana Dolores Krieger, nos anos de 1970, e passou a ser amplamente utilizado como prática de enfermagem. ${ }^{9}$

O toque terapêutico é uma terapia holística, ou seja, que considera o ser humano como um todo composto por partes - física, psíquica, espiritual - em constante interação, que não podem ser tratadas separadamente. A técnica consiste basicamente na imposição de mãos, com o intuito de harmonizar o campo de energia humano por meio do centramento do terapeuta, para posterior diagnóstico de quaisquer alterações, seguido da repadronização e da harmonização do campo de energia humano, conforme protocolado no North American Nursing Diagnostics Association (NANDA). ${ }^{9,10}$

Diversos estudos que avaliaram sua aplicação demonstram grande efetividade. Dentre os resultados obtidos, verificaramse: redução na dor não oncológica e crônica, e da fadiga; modificação nos parâmetros de sinais vitais, como pressão arterial, frequências cardíaca e respiratória, tanto em indivíduos em estado crítico, quanto em indivíduos saudáveis; e melhora nos padrões de sono. ${ }^{7,8,11,12}$

Técnicas como o toque terapêutico são de baixo custo, fácil aplicabilidade e podem atuar positivamente com relação aos sintomas físicos como os citados, bem como no controle de distúrbios do humor, estresse e/ou ansiedade. ${ }^{13,14}$ Diante disso, teve-se como questão norteadora desta pesquisa: Qual a eficácia do toque terapêutico como estratégia de redução de estresse vivenciado por estudantes de enfermagem?

Objetivou-se, desse modo, analisar o toque terapêutico como estratégia de redução de estresse em estudantes de enfermagem.

\section{MÉTODOS}

Estudo longitudinal, do tipo casocontrole, realizado com 20 estudantes de 
graduação em enfermagem de uma universidade pública da Região Sul do Brasil. Inicialmente, para a seleção dos 20 estudantes aplicou-se um questionário de caracterização e a escala Avaliação de Estresse em Estudantes de Enfermagem em 120 estudantes. Trata-se de uma escala autoaplicável que foi construída e validada no Brasil em estudo realizado com 160 estudantes de enfermagem de uma universidade de São Paulo. ${ }^{15}$

Os critérios de inclusão para os 120 estudantes foram ser estudante do curso de graduação em enfermagem da universidade do estudo, ser maior de 18 anos, manifestar interesse em participar da pesquisa, ter disponibilidade de horários para responder ao questionário de caracterização e a escala Avaliação de Estresse em Estudantes de Enfermagem e disponibilidade para participar das sessões de toque terapêutico. Estar grávida foi critério de exclusão.

A Avaliação de Estresse em Estudantes de Enfermagem apresenta uma escala do tipo Likert de quatro pontos, composta por 30 itens, agrupados em seis domínios: realização das atividades práticas (D1); comunicação profissional (D2); gerenciamento do tempo (D3); ambiente (D4); formação profissional (D5); e atividade teórica (D6). De acordo com os itens, o estudante marca com um " $\mathrm{X}$ " o número correspondente à intensidade de estresse que a situação lhe provoca, conforme a seguinte legenda: zero para "não vivencio a situação"; 1 para "não me sinto estressado com a situação"; 2 para "me sinto pouco estressado com a situação"; e 3 para "me sinto muito estressado com a situação". ${ }^{15}$

Para aferição do resultado, soma-se o número correspondente à intensidade de estresse dos itens presentes em cada domínio, sendo baixo, médio, alto ou muito alto, a saber: D1: zero a 9, nível baixo; 10 a 12, nível médio; 13 a 14, nível alto; 15 a 18, nível de estresse muito alto; D2: zero a 5, nível baixo; 6, nível médio; 7 a 8, nível alto; 9 a 12, nível de estresse muito alto; D3: zero a 10, nível baixo; 11 a 12, nível médio; 13 a 14, nível alto; 15, nível de estresse muito alto; D4: zero a 7, nível baixo; 8 a 10, nível médio; 11, nível alto; 12, nível de estresse muito alto; D5: zero a 9, nível baixo; 10, nível médio; 11 a 12, nível alto; 13 a 18, nível de estresse muito alto; D6: zero a 9, nível baixo; 10 a 11, nível médio; 12 a 13 nível alto; 14 a 15 , nível de estresse muito alto. ${ }^{15}$

A partir da análise dos 120 instrumentos, 76 estudantes de enfermagem apresentaram nível de estresse alto ou muito alto em um ou mais domínios da escala Avaliação de Estresse em Estudantes de Enfermagem. Destes, foram selecionados por meio de um sorteio 20 estudantes que participaram dos Grupos Controle e Experimental, sendo 10 para 
cada grupo. Utilizou-se, como parâmetro para definir o tamanho da amostra e sua forma de seleção, um estudo sobre as tendências da pesquisa envolvendo o uso de toque terapêutico como estratégia de enfermagem. $^{12}$

Os primeiros dez estudantes sorteados foram considerados Grupo Experimental e o segundo grupo de dez estudantes, Controle. Foram realizadas sete sessões de toque terapêutico com Grupo Experimental, com duração média de 30 minutos cada. Cabe ressaltar que, para essa prática de imposição de mãos, não existe um número fixo de sessões, nem tempo preestabelecido de duração. Recomenda-se apenas que o terapeuta considere os padrões do campo de energia humano do paciente para tal avaliação. ${ }^{9}$ A inexistência de protocolos pode ser considerada um fator limitante no que se refere à metodologia científica e reprodução de pesquisas, mas é compreensível, uma vez que cada indivíduo é único e precisa ser tratado como tal. ${ }^{16}$

As sessões de toque terapêutico ocorreram num Laboratório de Práticas de Enfermagem, com área física de $50 \mathrm{~m}^{2}$, pertencente à universidade em que este estudo foi realizado. Esse laboratório forneceu equipamentos para $\mathrm{o}$ reconhecimento e a verificação de sinais vitais, bem como macas para que os estudantes pudessem deitar-se confortavelmente para receber o toque terapêutico.

Cada sessão consistiu no preenchimento, pelo terapeuta, de uma ficha avaliativa adaptada, antes e após o recebimento do toque terapêutico, contendo diagnóstico do campo energético, sinais vitais (pressão arterial, temperatura axilar, frequências cardíaca e respiratória), saturação de oxigênio e mensuração do campo de energia humano, com a utilização de um aurímetro e fita métrica. Cabe ressaltar que se tratou de grupos não pareados. Ao término de todas as sessões de toque terapêutico, os estudantes dos Grupos Experimental e Controle responderam novamente a escala autoaplicável de Avaliação de Estresse em Estudantes de Enfermagem.

A coleta de dados foi realizada no período de abril a novembro de 2015 . Os dados foram submetidos à análise estatística descritiva, com frequência absoluta e relativa, para variáveis categóricas, e uso das medidas de tendência central (mediana), para variáveis numéricas, contando com o auxílio do software Statistical Package for Social Sciences (SPSS), versão 22.0. Realizaramse o teste dos postos com sinais de Wilcoxon, para verificar as diferenças entre os escores dos domínios da escala Avaliação de Estresse em Estudantes de Enfermagem, antes e após o recebimento 
do toque terapêutico, e o teste de KruskalWallis, para a comparação de medianas para as variáveis com mais de duas categorias. Em todos os testes, considerouse estatisticamente significante o valor de $\mathrm{p}<0,05$.

O desenvolvimento do estudo atendeu às normas nacionais $\mathrm{e}$ internacionais de ética em pesquisa envolvendo seres humanos, recebendo parecer favorável ao seu desenvolvimento do Comitê de Ética em Pesquisa com Seres Humanos da Área da Saúde da Universidade Federal do Rio Grande, sob o número 177/2014.

\section{RESULTADOS}

Cada grupo foi composto por dez estudantes de graduação em enfermagem. Dentre eles, $40 \%$ cursavam a oitava série, seguidos de $20 \%$ que cursavam a sexta série; e $90 \%$ eram do sexo feminino. A média de idade foi de 24,9, com mínimo de 18 e máximo de 37 anos.

Predominantemente, os estudantes se autorreferiram como da cor branca $(85 \%)$. Observaram-se menor frequência de casados (15\%) e predomínio de solteiros (85\%). Constatou-se que $30 \%$ possuíam renda familiar de até um salário mínimo (30\%), 40\% residiam com os pais e $10 \%$ tinham filhos. Em relação a ingerir bebidas alcoólicas, 50\% referiram fazê-lo; $15 \%$ eram fumantes; $25 \%$ afirmaram praticar atividade física; e $70 \%$ relataram possuir atividade de lazer.

Os estudantes, em sua maioria, não trabalhavam (70\%); dentre os que trabalhavam, 20\% já atuavam na área da saúde. Observou-se que $60 \%$ dos estudantes já tinham pensado em desistir do curso de graduação em enfermagem. Destaca-se que as sessões de toque terapêutico variaram de duas a sete, sendo a média de 5,2 , visto que nem todos os estudantes puderam comparecer a todas as sessões.

Na Tabela 1, consta a mediana da escala Avaliação de Estresse em Estudantes de Enfermagem antes e após o recebimento do toque terapêutico, tanto no Grupo Experimental, quanto no Controle. 
Tabela 1: Escala Avaliação de Estresse em Estudantes de Enfermagem, antes e após o recebimento do toque terapêutico $(n=20)$

\begin{tabular}{ccccc}
\hline Domínio & \multicolumn{2}{c}{ Grupo Experimental $(\mathbf{n}=\mathbf{1 0})$} & \multicolumn{2}{c}{ Grupo Controle $(\mathbf{n}=\mathbf{1 0})$} \\
\hline & Antes & Após & Antes & Após \\
\hline 1 & 14,00 & 8,00 & 12,50 & 9,00 \\
\hline 2 & 7,00 & 5,50 & 6,00 & 6,50 \\
\hline 3 & 12,50 & 9,50 & 11,50 & 11,50 \\
\hline 4 & 3,50 & 3,00 & 7,50 & 6,00 \\
\hline 5 & 12,00 & 10,50 & 12,00 & 11,50 \\
\hline 6 & 12,00 & 8,50 & 9,50 & 9,50 \\
\hline
\end{tabular}

Resultados expressos em mediana.

Na Tabela 2, apresenta-se o teste de Wilcoxon com as diferenças entre os escores dos domínios da escala Avaliação de Estresse em Estudantes de Enfermagem após o recebimento do toque terapêutico. O toque terapêutico auxiliou na redução do estresse vivenciado por estudantes de enfermagem do grupo Experimental em relação aos domínios 1,3 e 6 da escala Avaliação de Estresse em Estudantes de Enfermagem (D1 - realização das atividades práticas; D3 - gerenciamento do tempo; e D6 - atividade teórica).

Tabela 2: Diferenças entre os escores dos domínios da escala Avaliação de Estresse em Estudantes de Enfermagem após o recebimento do toque terapêutico $(n=20)$

\begin{tabular}{ccc}
\hline Domínio & Grupo Experimental $(\mathbf{n}=\mathbf{1 0})$ & Grupo Controle $(\mathbf{n}=\mathbf{1 0})$ \\
\hline 1 & $\mathrm{Z}=-2,604^{*} ; \mathrm{p}=0,009$ & $\mathrm{Z}=-1,611^{*} ; \mathrm{p}=0,107$ \\
\hline 2 & $\mathrm{Z}=-1,866^{*} ; \mathrm{p}=0,062$ & $\mathrm{Z}=-1,732 \dagger ; \mathrm{p}=0,083$ \\
\hline 3 & $\mathrm{Z}=-2,099^{*} ; \mathrm{p}=0,032$ & $\mathrm{Z}=-, 514 \uparrow ; \mathrm{p}=0,607$ \\
\hline 4 & $\mathrm{Z}=-1,065^{*} ; \mathrm{p}=0,287$ & $\mathrm{Z}=-, 281^{*} ; \mathrm{p}=0,779$ \\
\hline 5 & $\mathrm{Z}=-1,554^{*} ; \mathrm{p}=0,120$ & $\mathrm{Z}=-, 35 \boldsymbol{1}^{\dagger} ; \mathrm{p}=0,722$ \\
\hline 6 & $\mathrm{Z}=-2,699^{*} ; \mathrm{p}=0,007$ & $\mathrm{Z}=-, 493^{*} ; \mathrm{p}=0,622$ \\
\hline
\end{tabular}

*Baseado em ranks positivos; $\uparrow$ Baseado em ranks negativos.

A Tabela 3 refere-se à associação entre os domínios 1, 3 e 6 da escala Avaliação de Estresse em Estudantes de Enfermagem e atividade de lazer, renda, ter ou não filhos e série atual.

Tabela 3: Associação entre os domínios da escala Avaliação de Estresse em Estudantes de Enfermagem e as atividades de lazer, a renda, o fato de ter ou não filhos e a série atual $(n=20)$

\begin{tabular}{cccccc}
\hline Domínio & Variáveis & Grupo Experimental $(\mathbf{n}=\mathbf{1 0})$ & \multicolumn{2}{c}{ Grupo Controle $(\mathbf{n}=\mathbf{1 0})$} \\
\hline \multirow{3}{*}{1} & Atividade de lazer & $1,72^{*}$ & $\mathrm{p}=0,19$ & $0,69^{*}$ & $\mathrm{p}=0,79$ \\
\cline { 2 - 6 } & Renda & $3,82^{*}$ & $\mathrm{p}=0,57$ & $6,33^{*}$ & $\mathrm{p}=0,17$ \\
\cline { 2 - 6 } & Filhos & $0,07^{*}$ & $\mathrm{p} 0,78$ & $-*$ & - \\
\cline { 2 - 6 } & Série atual & $3,66^{*}$ & $\mathrm{p}=, 30$ & $6,34^{*}$ & $\mathrm{p}=0,17$ \\
\hline \multirow{3}{*}{3} & Atividade de lazer & $0,42^{*}$ & $\mathrm{p}=0,51$ & $0,28^{*}$ & $\mathrm{p}=0,59$ \\
\cline { 2 - 6 } & Renda & $4,28^{*}$ & $\mathrm{p}=0,50$ & $4,56^{*}$ & $\mathrm{p}=0,33$ \\
\cline { 2 - 6 } & Filhos & $0,01^{*}$ & $\mathrm{p}=0,89$ & - - $^{*}$ & - \\
\cline { 2 - 6 } & Série atual & $2,89^{*}$ & $\mathrm{p}=0,40$ & $5,84^{*}$ & $\mathrm{p}=0,21$ \\
\hline
\end{tabular}




\begin{tabular}{cccccc}
\hline \multirow{4}{*}{6} & Atividade de lazer & $0,05^{*}$ & $\mathrm{p}=0,82$ & $3,46^{*}$ & $\mathrm{p}=0,63$ \\
\cline { 2 - 6 } & Renda & $2,71^{*}$ & $\mathrm{p}=0,74$ & $4,79^{*}$ & $\mathrm{p}=0,30$ \\
\cline { 2 - 6 } & Filhos & $1,24^{*}$ & $\mathrm{p}=0,26$ & $-*$ & - \\
\cline { 2 - 6 } & Série atual & $2,73^{*}$ & $\mathrm{p}=0,43$ & $7,71^{*}$ & $\mathrm{p}=0,10$ \\
\hline
\end{tabular}

*Kruskal-Wallis.

$\mathrm{Na}$ amostra estudada, a diferença entre o número de sessões recebidas e as variáveis atividade de lazer, renda e série atual não interferiu nos níveis de estresse dos estudantes de nenhum dos dois grupos, segundo o teste de Kruskal-Wallis. A variável ter ou não filhos não interferiu no nível de estresse dos estudantes do Grupo Experimental. No Grupo Controle, nenhum dos estudantes tinha filho.

\section{DISCUSSÃO}

O toque terapêutico mostrou-se estratégia eficaz de redução de estresse em estudantes de enfermagem em relação à realização das atividades práticas, gerenciamento do tempo e atividade teórica. O toque terapêutico tem como principal característica a capacidade de promover o relaxamento tanto do terapeuta, quanto do receptor, além do desenvolvimento da consciência do autocuidado, o que é extremamente positivo para o enfrentamento do estresse. $^{17}$ Em revisão integrativa publicada em 2015, de dez artigos que analisavam o toque terapêutico aplicado ao cuidado de estresse-ansiedade, oito obtiveram resultados estatisticamente significativos. $^{17}$

As atividades práticas constituem um dos fatores responsáveis pelo acometimento de estresse nos estudantes, mas foi possível perceber a efetividade do toque terapêutico quanto a tal assunto nas questões especificadas no domínio 1, que versam sobre as atividades práticas. O estágio supervisionado, em especial, é apontado como grande gerador de estresse por parte dos estudantes, uma vez que os mesmos saem de uma situação ideal descrita pela teoria e se deparam com a realidade da prática clínica sem estarem aparentemente preparados para tal vivência. ${ }^{18-20}$

O domínio 3 aborda questões relacionadas ao gerenciamento de tempo. As exigências de atividades acadêmicas, muitas vezes, vêm acompanhadas de um afastamento da família e do grupo de amigos, podendo intensificar no estudante os sentimentos de solidão, abandono e isolamento. ${ }^{18,21}$ Por outro lado, se o mesmo for capaz de utilizar estratégias de enfrentamento eficazes, pode perceber essa experiência de forma positiva, possibilitando maior autonomia e capacidade de adaptação. ${ }^{6,21}$ Para tanto, faz-se necessário instrumentalizar o 
estudante, e o toque terapêutico pode ser utilizado como uma estratégia eficaz.

Os estudantes de enfermagem apresentam manifestações psicológicas e fisiológicas de estresse em maior proporção, se comparados aos de outras áreas. $^{6}$ A identificação dos fatores desencadeadores de estresse por parte desses estudantes é primordial e, para tanto, é necessário que eles sejam capazes de desenvolver o autoconhecimento, refletindo sobre suas ações e sentimentos. ${ }^{17}$ É de suma importância capacitar os docentes para identificar sinais sugestivos de estresse e ansiedade, bem como instrumentalizar a academia para atuar frente aos estressores, amenizando-os quando possível e auxiliando os estudantes de enfermagem para enfrentá-los, pois a maneira com que este estudante é inserido em sua área de atuação pode diferir entre uma vivência frustrante e um trabalho prazeroso. $^{3,19,21}$

De acordo com diversas pesquisas, as atividades teóricas, em especial as avaliações, têm se mostrado como algumas das atividades mais estressantes para os estudantes de enfermagem. ${ }^{19}$ Segundo os próprios estudantes, os sistemas de avaliação são desgastantes e causam sobrecarga de trabalho, o que acarreta em menor tempo para realizar outras atividades. ${ }^{1,6} \mathrm{O}$ toque terapêutico também se mostra estatisticamente positivo no que se refere à diminuição dos níveis de estresse dos estudantes no domínio que engloba questões relacionadas às atividades teóricas (D6), o que é de grande valia, uma vez que o estresse, frente à avaliação pode paralisar o estudante, levando-o à dificuldade de concentração, por exemplo.

As pesquisas envolvendo o toque terapêutico vêm ganhando forças, especialmente na última década. Porém, a ausência de um protocolo é considerada um fator ainda limitante pela academia, o que acaba retardando o uso do toque terapêutico, tanto como uma estratégia de enfrentamento de estresse, quanto de dor, ansiedade e distúrbios do sono. ${ }^{15}$ Pesquisas sobre a imposição de mãos são recentes dentro de um contexto universitário[,] embora a realização dessa prática seja milenar.

As limitações deste estudo estiveram relacionadas ao pequeno número de participantes. Tal fato ocorreu em função de falta de uma estrutura física que comportasse o atendimento de mais estudantes concomitantemente para receber o toque terapêutico e pela inexistência de mais estudantes terapeutas habilitados para a aplicação. Outras variáveis foram testadas a fim de verificar se estavam ou não interferindo no nível de estresse dos estudantes. Contudo, nenhuma dessas variáveis interferiu no nível de 
estresse dos estudantes do Grupo

Experimental e no Grupo Controle.

\section{CONCLUSÃO E CONTRIBUIÇÕES}

$\mathrm{O}$ toque terapêutico se mostrou estatisticamente eficaz para a redução de estresse em estudantes de enfermagem em três dos seis domínios avaliados (estresse relacionado às atividades prática, ao tempo e às atividades teóricas). O presente estudo demonstrou a importância de aprofundar pesquisas na área que visem propiciar recursos que os estudantes de enfermagem possam buscar a fim de minimizar o estresse vivenciado. No entanto, fatores limitadores, como o pequeno número de sujeitos, impossibilitam a generalização dos resultados.

\section{REFERÊNCIAS}

1. Hirsch CD, Barlem EL, Almeida LK, Tomaschewski-Barlem JG, Figueira AB, Lunardi VL. Coping strategies of nursing students for dealing with university stress. Rev Bras Enferm. [Internet]. 2015[citado em 01 abr 2018]; 68(5):783-90. Disponível em:

http://www.scielo.br/scielo.php?pid=S0034 $-71672015000500783 \&$ script $=$ sci_arttext

2. Del Prato D, Bankert E, Grust P, Joseph

J. Transforming nursing education: a review os stressors and estrategies that support students' professional socialization. Adv Med Educ Pract. [Internet]. 2011[citado em 01 abr 2018]; 2(1):109-16. Disponível em: https://www.ncbi.nlm.nih.gov/pmc/articles/
PMC3661250/

3. Chipas A, Cordrey D, Floyd D, Grubbs L, Miller S, Tyre B. Stress: perceptions, manifestations, and coping mechanisms of student registered nurse anesthetists. AANA J. 2012; 80(4 Suppl):49-55.

4. Kestenberg CC, Silva AV, Fabri JM, Silva NA, Rosa BM, Branco LM. Estresse em graduando de enfermagem: técnicas de relaxamento para lidar com fatores estressores. Interagir: Pensando a Extensão [Internet]. 2014[citado em 01 abr 2018]; 17/18/19: 37-43. Disponível em:

https://www.e-

publicacoes.uerj.br/index.php/interagir/arti cle/view/13565/10375

5. Esperidião E, Barbosa JA, Silva NS, Munari DB. A saúde mental do aluno de Enfermagem: revisão integrativa da literatura. SMAD, Rev Eletrônica Saúde Mental Álcool Drog [Internet].

2013 [citado em 01 abr 2018]; 9(3): 14453. Disponível em:

http://www.revistas.usp.br/smad/article/vie w/86745

6. Labrague L, McEnroe-Petitte DM, Papathanasiou IV, Edet OB, Tsaras K, et al. A literature review on stress and coping strategies in nursing students. J Ment Health. [Internet]. 2017[citado em 05 set 2018]; 26(5):471-80. Disponível em: https://www.tandfonline.com/doi/pdf/10.10 80/09638237.2016.1244721

7. Mello TC, Brito RS. Efetividade do toque terapêutico no alívio de sintomatologia do paciente. Saúde (Santa Maria) [Internet]. 2015[citado em $21 \mathrm{abr}$ 2018]; 41(2):45-52. Disponível em: http://cascavel.ufsm.br/revistas/ojs2.2.2/index.php/revistasaude/article/view/1 4472

8. Conselho Federal de Enfermagem (Brasil). Resolução COFEN-389/2011. Atualiza, no âmbito do Sistema Cofen/Conselhos Regionais de Enfermagem, os procedimentos para registro de título de pós-graduação lato e stricto sensu concedido a Enfermeiros e lista as Especialidades [Internet]. Brasília, DF: COFEN; 2011 [citado em 01 abr 
2018]. Disponível em:

http://www.cofen.gov.br/resoluo-cofen-n3892011_8036.html

9. Krieger D. Therapeutic touch inner workbook. Santa Fe: Bear \& Company; 1997.

10. Diagnósticos de Enfermagem da NANDA: definições e classificação 20122014. Garcez, RM, Tradutora. Porto Alegre: Artmed; 2013.

11. Marta IE, Baldan SS, Berton AF, Pavan M, Silva MJ. The effectiveness of therapeutic touch on pain, depression and sleep in patients with chronic pain: clinical trial. Rev Esc Enferm USP. [Internet]. 2010 [citado em 01 abr 2018]; 44(4):11006. Disponível em: http://www.scielo.br/scielo.php?script=sci_ arttext\&pid=S0080-

$62342010000400035 \& \operatorname{lng}=\mathrm{en} \& \mathrm{nrm}=\mathrm{iso} \& \mathrm{t}$ $\operatorname{lng}=\mathrm{en}$

12. Vasquez CI, Santos DS, Carvalho EC. Tendências da pesquisa envolvendo o uso do toque terapêutico como uma estratégia de enfermagem. Acta Paul Enferm. [Internet]. 2011 [citado em 01 abr 2018]; 24(5):712-4. Disponível em:

http://www.scielo.br/scielo.php?script=sci_ arttext\&pid=S0103-

$21002011000500019 \& \operatorname{lng}=\mathrm{en} \& \mathrm{nrm}=\mathrm{iso} \& \mathrm{t}$ $\operatorname{lng}=\mathrm{pt} \&$ ORIGINALLANG $=\mathrm{pt}$

13. Nima AA, Rosenberg P, Archer T,

Garcia D. Anxiety, affect, self-esteem, and stress: mediation and moderation effects on depression. PLoS ONE. [Internet].

2013[citado em 01 abr 2018]; 8(9):

e73265. Disponível em:

http://journals.plos.org/plosone/article?id= 10.1371/journal.pone.0073265

14. Thomas LS, Stephenson N, Swanson

M, Jesse DE, Brown S. A pilot study: the effect of healing touch on anxiety, stress, pain, pain medication usage, and physiological measures in hospitalized sickle cell disease adults experiencing a vaso-occlusive pain episode. J Holist Nurs. [Internet]. 2013 [citado em 01 abr 2018]; 20(10): 234-47. Disponível em: http://journals.sagepub.com/doi/pdf/10.117 7/0898010113491631
15. Costa AL, Polak C. Construção e validação de instrumento para Avaliação de Estresse em Estudantes de Enfermagem (AEEE). Rev Esc Enferm USP. [Internet]. 2009 [citado em 01 abr 2018]; 43(n esp):1017-26. Disponível em: http://www.scielo.br/scielo.php?script=sci_ arttext\&pid=S0080-62342009000500005 16. Souza AL, Prado BO, Silva RP, Chaves EC, Lunes DH. Clinical research with therapeutic touch: a systematic review. Sci Med. [Internet]. 2014 [citado em $01 \mathrm{abr}$ 2018]; 24(4):404-10. Disponível em: https://dialnet.unirioja.es/servlet/articulo?c odigo $=5661898$

17. Motta PM, Barros NF. A aplicação de técnicas de imposição de mãos no estresseansiedade: revisão sistemática da literatura. Cad Ter Ocup UFSCar. [Internet]. 2015[citado em 01 abr 2018]; 23(2):38192. Disponível em:

http://www.cadernosdeterapiaocupacional. ufscar.br/index.php/cadernos/article/view/1 147

18. Silva VL, Chiquito NC, Andrade RA, Brito MF, Camelo SH. Estressores entre acadêmicos de enfermagem de uma universidade pública. Rev Enferm UERJ. [Internet]. 2011 [citado em 01 abr 2018]; 19(1):121-6. Disponível em: http://www.epublicacoes.uerj.br/index.php/enfermagem uerj/article/view/5992

19. Tomaschewski-Barlem JG, Lunardi VL, Ramos AM, Silveira RS, Barlem EL, Ernandes CM. Manifestações da síndrome de burnout entre estudantes de graduação em enfermagem. Texto \& Contexto Enferm. [Internet]. 2013 [citado em 01 abr 2018]; 22(3): 754-62. Disponível em: http://www.scielo.br/scielo.php?script=sci arttext\&pid=S0104-

$07072013000300023 \& \operatorname{lng}=\mathrm{en} \& \mathrm{nrm}=\mathrm{iso} \& \mathrm{t}$ lng $=$ pt\&ORIGINALLANG $=\mathrm{pt}$

20. Edwards D, Burnard P, Bennett K, Hebden U. A longitudinal study of stress and self-esteem in student nurses. Nurse Educ Today. [Internet]. 2010 [citado em 01 abr 2018]; 30(1):78-84. Disponível em: https://www.sciencedirect.com/science/arti cle/pii/S0260691709001208 
21. Shdaifat E, JamamaA, Al-Amer M.

Stress and coping strategies among nursing students. Glob J Health Sci. [Internet].

2018 [citado em 05 set 2018]; 10(5): 33-

41. Disponível em:

https://www.researchgate.net/publication/3

23869280/download

RECEBIDO: $24 / 06 / 2018$

APROVADO: $18 / 10 / 2018$

PUBLICADO: $12 / 2018$ 